\title{
Pathological Changes in the Autologous Pericardial Aortic Valve at 10 Years
}

\author{
Sachin Talwar ${ }^{1 *}$, Neeti Makhija², Ira Dhawan², Arkalgud Sampath Kumar ${ }^{3}$ and Sukhjeet Singh \\ ${ }^{1}$ Department of Cadiothoracic and Vascular surgery, All India Institute of Medical, India \\ ${ }^{2}$ Department of Cardiac Anesthesia, All India Institute of Medical, India
}

${ }^{3}$ Formerly Professor and Head and Chief Cardiothoracic Centre, All India Institute of Medical Sciences, India

Received: September 05, 2017; Published: September 20, 2017

*Corresponding author: Sachin Talwar, Professor, Department of Cardio Thoracic and Vascular Surgery, All India Institute of Medical Sciences, New Delhi-110029, India; Tel- 911126594835; Fax- 911126588663; Email: sachintalwar@hotmail.com

\begin{abstract}
A 41-year old patient who underwent a successful re-operation 10 years following aortic valve replacement with autologous pericardium is reported. The findings at re-operation are discussed.
\end{abstract}

Keywords: Aortic valve replacement; Duran Valve; Re-operation.

Abbreviation: mAVR: Mechanical Valve; ASK: Senior Author; AS: Aortic Stenosis; AI: Aortic Insufficiency; CPB: Cardiopulmonary Bypass

\section{Introduction}

Aortic valve replacement with a stentless autologous pericardial valve was proposed by Duran and colleagues to avoid complications of a Mechanical Valve (mAVR). The procedure has been demonstrated to be an inexpensive substitute to mAVR and avoids long term costs and compliance associated with anticoagulation, more so in the third world [1,2]. Acceptable midterm and long term results have been reported [2-5]. However, it is not expected to be a permanent substitute because the pericardium is expected to undergo degenerative changes in the mid to long term. Therefore, it is essential for the surgeons to be aware of what to expect at re-operation and be prepared to perform a safe re-operation. We recently successfully re-operated one such patient who had undergone pericardial AVR and report the findings as well as the technique of re-operation.

\section{Case Report}

Our institutional ethics committee waived the need for a formal ethics committee approval and the patient gave consent to publish this report and images. A 41 year old male patient presented with dyspnea on exertion NYHA class II of six months duration. Ten years ago, he was operated upon by the Senior Author (ASK). At that time, the native aortic valve leaflets were excised and the aortic valve annulus was sized using special Duran's valve seizers. A strip of pericardium was cut using specially designed moulds and the pericardial strip was buttressed between two snugly fitting parts of that mould. This was immersed in $0.625 \%$ glutaraldehyde for
10 minutes and was later rinsed in normal saline. The resultant trileaflet pericardial valve was sutured inside the aorta using the technique [1].

He continued to be off medication and in NYHA class II with the last echocardiogram 2 years ago showing thickening of the neo aortic valve leaflets with mild to moderate Aortic Stenosis (AS) with a peak systolic gradient of $40 \mathrm{~mm} \mathrm{Hg}$ and mild Aortic Insufficiency (AI). His symptoms deteriorated since then and most recent echocardiography revealed severe calcific AS, a peak systolic gradient of $128 \mathrm{~mm} \mathrm{Hg}$ with normal biventricular function. Coronary angiography revealed no coronary artery disease. At reoperation via a sternotomy, the dense adhesions were lysed. Intra operative TEE demonstrated heavily calcified tricuspid valve leaflets with severe AS and moderate AI (Figure 1). Local CO2 insufflations were used for deairing, thus avoiding complete mobilization of the heart due to dense adhesions. Aorto bicaval cannulation was performed and mildly hypothermic Cardiopulmonary bypass (CPB) $\left(32^{\circ} \mathrm{C}\right)$ was established.

The pulmonary artery was vented. Aorta was cross clamped, and after aortotomy, a single dose of Delnido cardioplegia was delivered directly into the coronary Ostia. The leaflets of the pericardial valve were heavily calcified with calcification extending on to the mitral valve leaflets and the aortic wall (Figure 2). However, the tricuspid architecture of the pericardial valve that had been fashioned out of the native pericardium was well preserved. This valve was excised 
taking care not to damage the mitral valve and aortic wall while protecting the coronary ostia from dislodged calcium debris. This took nearly 30 minutes and the technique was no different from that used to excise a heavily calcified native stenotic aortic valve.

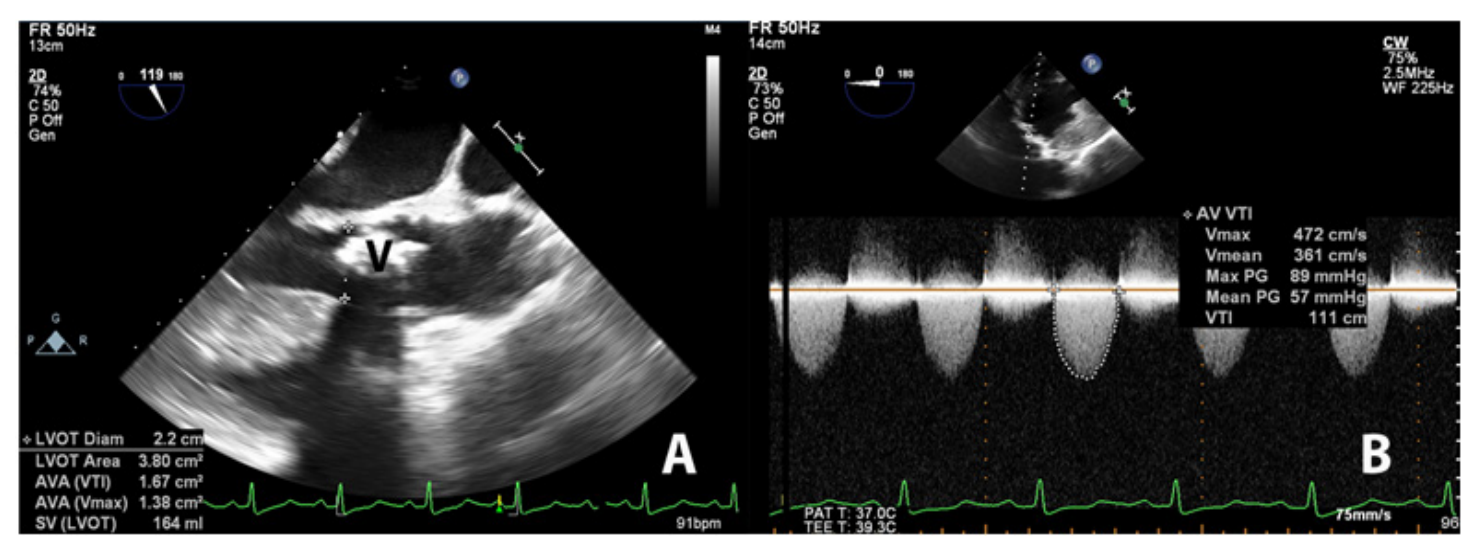

Figure 1: Intra operative transesophageal echocardiography.

A. Two-dimensional mid-esophageal aortic valve long-axis view showing severely calcified aortic valve leaflets (V).

B. Two-dimensional deep transgastric long-axis view showing evidence of severe aortic stenosis.

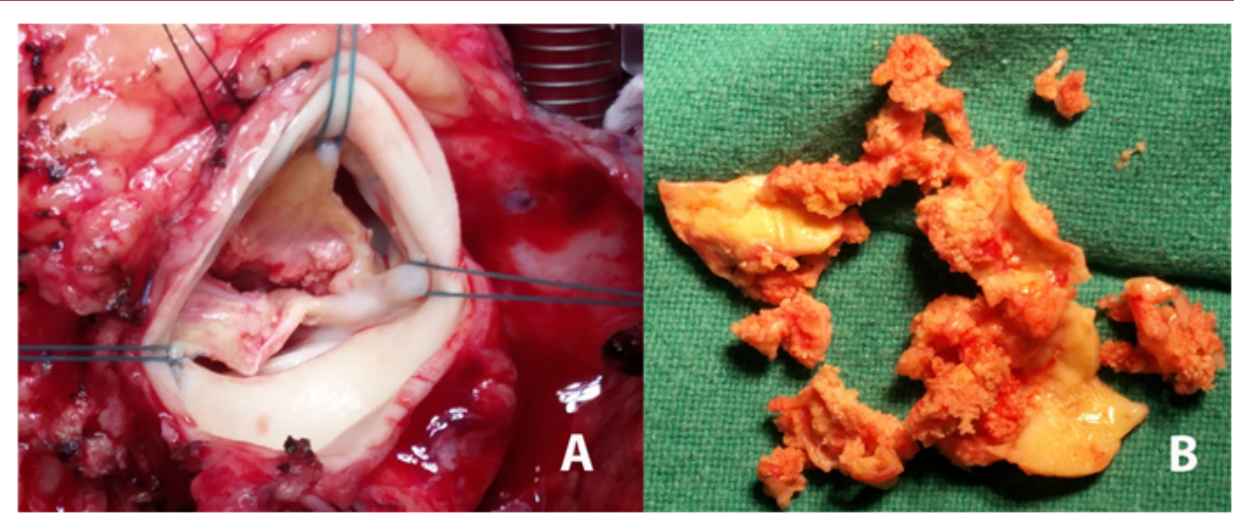

\section{Figure 2:}

A. Intaoperative image shows the heavily calcified aortic valve before excision.

B. Intaoperative image shows the heavily calcified aortic valve after excision.

Following this, the aortic valve was replaced with a $24 \mathrm{MM}$ mechanical Medtronic ATS open pivot TM heart valve (Medtronic Inc., Medtronic Parkway, MN, US). The aortotomy was closed and the patient was uneventfully weaned off CPB without any inotropic support. CPB time was 116 minutes and the Aortic cross clamp time was 68 minutes. Post-operative recovery was uneventful and the patient was discharged home on 6th post operative day on oral anticoagulants, diuretics and beta blockers. Histopathology examination of the excised valve showed dystrophic calcification with no viable tissue. There was no evidence of any immunologic response to the pericardial leaflets as evidenced by complete lack of inflammatory infiltrates. Thus, the findings were suggestive of pure degeneration and calcification of the pericardial leaflets.

\section{Discussion}

AVR with Duran pericardial valve was proposed as an alternative to Mavr [1]. Besides superior hemodynamics compared to a mechanical valve, it is more resistant to infection and avoids the long term hazards of anticoagulation [6]. Excellent mid and long-term results have been reported [2-5]. However, all patients undergoing this operation are expected to require a re-operation due to calcification and structural deterioration of the valve [3]. Data on re-operations on such patients has only discussed the need for re-operation without detailing the findings and no published images are available. We provide such images in this report.

The precise mechanism of calcification and degeneration of these valves is unknown, but immune responses and mechanical shear stresses have been implicated [7,8]. Immune responses are more likely to play a role in the degeneration of heterologous rather than autologous tissues. In the case of the latter, degenerative calcification resulting from abnormal shear stresses on the non pliable leaflets appears to play a more decisive role. Glutaryldehyde treatment of the autologous pericardium is essential to maintain the shape and symmetry of the reconstructed pericardial valve. However, glutaryldehyde treatment of the pericardium per se is 
a necessary evil and leads to alterations in collagen linkages and architecture which favors degenerative processes and calcification, that can often be very dense, as in the present patient.

Long term survival following mechanical AVR is $59 \% \pm 2 \%$ at 10 years [9]. In a long-term study of patients undergoing mAVR, the linear zed rates per 100 patients years of follow-up were embolism 0.25 , paravalvular leakage 0.25 , prosthetic endocarditic 0.25 and reoperation 0.50 [10]. This compares favorably with the results of pericardial AVR [2-4]. Despite a reoperation at 10 years, our patient was in NYHA class I/II at follow up and the reoperation was safe. This confirms that with meticulous initial technique, long term results are satisfactory. However, the reoperation can be technically demanding due to densely calcified valve leaflets and utmost care needs to be exercised.

\section{Compliance with Ethical Standards}

\section{Funding}

The study did not receive any funding.

\section{Statement of Human rights/Ethical Approval}

All procedures performed in this study were in accordance with the ethical standards of the institutional and national research committee and with the 1964 Helsinki declaration and its later amendments or comparable ethical standards. For this study, formal consent was obtained.

\section{Informed Consent}

Informed consent was obtained from all individual participants included in the study.

\section{References}

1. Duran CM, Gometza B, Kumar N, Gallo R, Martin Duran R (1995) Aortic valve replacement with freehand autologous pericardium. J Thorac Cardiovasc Surg 110(2): 511-516.

2. Goetz WA, Lim HS, Lansac E, Weber PA, Duran CM (2002) A temporarily stented, autologous pericardial aortic valve prosthesis. J Heart Valve Dis 11(5): 696-702.

3. Al Halees Z, Al Shahid M, Al Sanei A, Sallehuddin A, Duran C (2005) Up to 16 years follow-up of aortic valve reconstruction with pericardium: a stentless readily available cheap valve. Eur J Cardiothorac Surg 28(2): 200-205.

4. Mittal CM, Talwar S, Velayoudham D, Kothari SS, Kumar AS (2009) Early results of aortic valve reconstruction with stentless glutaraldehyde treated autologous pericardial valve. Indian J Thorac Cardiovasc Surg 25(4): 175-182.

5. Duran CM, Gometza B (1982) Aortic valve reconstruction in the young. J Card Surg 9(2): 204-208.

6. Edmunds LH Jr (1982) Thromboembolic complications of current cardiac valvular prostheses. Ann Thorac Surg 34: 96-106.

7. Grabenwoger M, Fitzal F, Gross D, Hutschala D, Bock P, et al. (2009) Different modes of degeneration in autologous and heterologous heart prostheses. J Heart Valve Dis 9(1): 104-109.

8. Chan KM, Rahman Haley S, Mittal TK, Gavino JA, Dreyfus G (2011) Truly stentless autologous pericardial aortic valve replacement: an alternative to standard aortic valve replacement. J Thorac Cardiovasc Surg 141(1): 276-283.

9. Ikonomidis JS, Kratz JM, Crumbley AJ, Stroud MR, Bradley SM, et al. (2003) Twenty-year experience with the St Jude Medical mechanical valve prosthesis. J Thorac Cardiovasc Surg 126(6): 2022-2031.

10. Aagaard J, Tingleff J, Andersen PV, Hansen CN (2003) Fourteen years' experience with the CarboMedics valve in young adults with aortic valve disease. J Heart Valve Dis 12(1): 81-86.

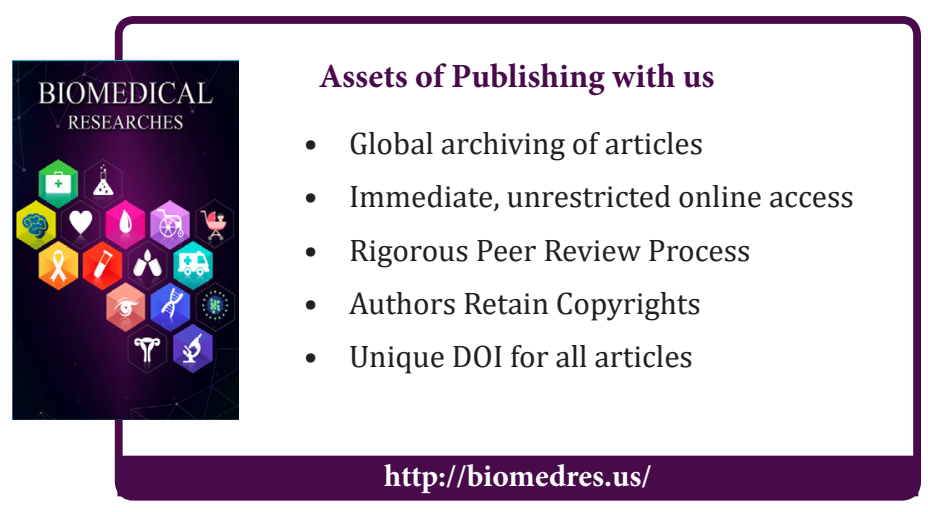

